\title{
芳基、烷基磷酸混酯类化合物的合成方法研究进展
}

\author{
焦林郁* 于 华宁资慧李卓 \\ (西北大学化工学院 西安 710069)
}

\begin{abstract}
摘要 芳基、烷基磷酸混酯是一类重要的有机磷化物, 广泛应用于生物、医药、农药、高分子以及材料科学等众多领 域，具有广阔的发展前景. 含磷基团所特有的物理、化学以及生物性质，为其结构修饰、衍生化以及应用领域的拓展提 供了更多可能，因此，该类化合物合成方法的研究具有重要的理论和现实意义. 综述了过去几十年芳基、烷基磷酸混酯 化合物制备方法的研究进展, 列举了其应用实例, 分类总结了多种不同类型的含磷试剂向目标化合物转化的实验方法 和发展历程，选择性地探讨了反应的特点、机理以及在合成中的应用，希望该综述能够为磷酸混酯类化合物新型制备 方法的开发提供参考, 为相关领域的学者、研究生以及其他人员提供一些有益借鉴.
\end{abstract}

关键词 合成方法; 磷酸混酯; 有机磷化物

\section{Research Progress in the Preparation of Aryl and Alkyl Mixed Phosphates}

\author{
Jiao, Linyu* Yu, Hua $\quad$ Ning, Zihui $\quad$ Li, Zhuo \\ (School of Chemical Engineering, Northwest University, Xi'an 710069)
}

\begin{abstract}
Aryl and alkyl mixed phosphates are very important phosphorus containing organic compounds, which are widely used in many fields, such as biology, medicine, pesticide, polymer, and material science. The unique physical, chemical, and biological properties of phosphorus-based motifs provide more possibilities for their structural modification, derivatization, and potential application. Therefore, the research on the preparation of these compounds has important theoretical and practical significance. In this paper, the research progress of the synthesis methodologies of aryl and alkyl mixed phosphate in the past few decades is reviewed. The application examples are listed. The experimental methods and development process of the conversion of various kinds of phosphorus containing reagents to target compounds are summarized. The characteristics, mechanism, and application of the reaction are discussed selectively as well. We hope that this review can provide a reference for the development of new preparation protocols of mixed phosphate, and provide some useful references for scholars, graduate students, and others in the related fields.
\end{abstract}

Keywords synthetic method; mixed phosphate; organic phosphorus compound

磷酸混酯是指磷酸与两种或者两种以上的羟基化 合物的酯化产物，是由磷中心原子及其外围的一个磷氧 双键和三个磷氧单键构成的四配位共价分子, 当参与成 键的差基试剂同时含有醇类和酚类化合物时，又被称为 芳基、烷基磷酸混酯 ${ }^{[1]}$. 作为一类重要的有机磷化物, 磷 酸混酯化合物广泛存在于自然界和功能分子中, 磷原子 固有的理化性质, 包括可极化性、多重氧化价态及配位 能力, 吸引着众多的化学家和材料科学家参与到其合 成、衍生化以及性质研究中. 研究表明, 对于有机分子 骨架来讲, 磷酸基团的引入可以显著改善母体分子的物
理和化学性质, 提高其化学反应性能和生物活性, 为后 续的结构修饰、衍生化以及应用提供更多可能. 磷酸混 酯化合物与人们的生产生活密切相关, 截至目前, 在包 括生物、医药、农药、高分子以及材料科学等众多领域 中应用广泛，特别是其优异的生物活性、润滑性以及阻 燃性能, 使其成为各自领域的研究热点和卓越代表.

如图 1 所示, 首先, 芳基、烷基磷酸混酯的较好的 生物活性使其广泛应用于农业领域，其中对硝基苯基二 乙基磷酸酯又称为对氧磷(Paraoxon), 是一种商业化的 防治农作物病虫害的农药 ${ }^{[2]}$. 当将其上的对硝基苯基替

* Corresponding author. E-mail: lyjiao@nwu.edu.cn

Received May 31, 2021; revised June 30, 2021; published online July 23, 2021.

Project supported by the National Natural Science Foundation of China (No. 21901150).

国家自然科学基金(No. 21901150)资助项目. 
换为 6-香豆素骨架, 通过特定位点的衍生化卤代反应, 可以分别得到杀虫剂 Coroxon 和神经毒剂 Depfmu(图 $1)^{[3]}$.
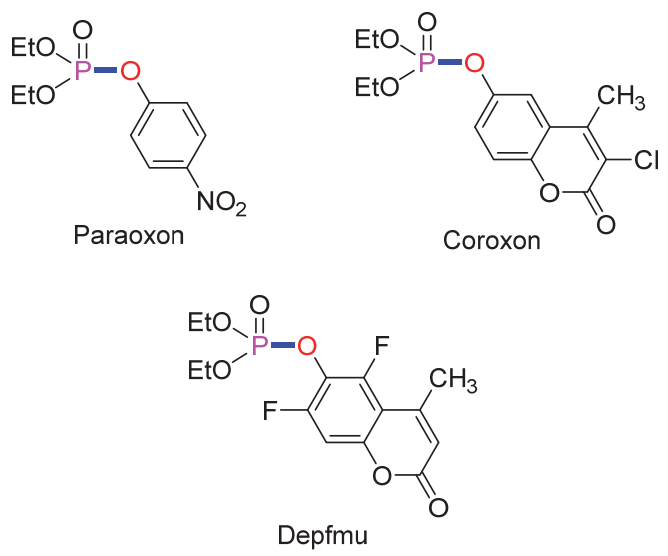

图 1 芳基、烷基磷酸混酯在农业方面的应用

Figure 1 Application of aryl and alkyl mixed phosphates in agriculture

其次, 磷酸混酯在生物和医药领域也有重要应用, 当脂肪链为含有一个双键的长链烷基时，该化合物可以 作为脂质的前体药物(图 2) ${ }^{[4]}$, 而对于如图 3 所示不同结 构的磷酸混酯, 从左到右依次为新型的具有第二信使功 能的分子骨架 ${ }^{[5]}$ 、抗肿瘤前药 ${ }^{[6]}$ 以及核苷酸药物前体 ${ }^{[7]}$.

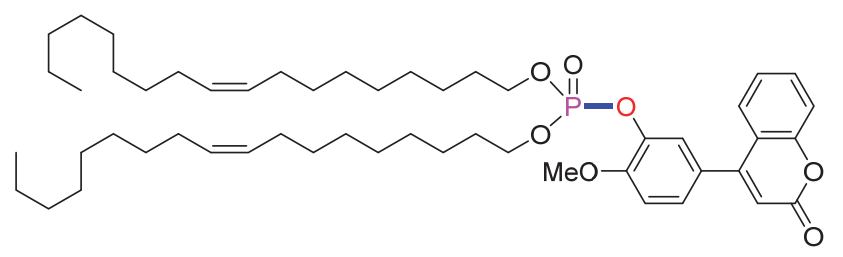

图 2 磷酸混酯类脂质前体药物

Figure 2 Aryl and alkyl mixed phosphates as lipid prodrug

再次, 磷酸混酯化合物也可应用于阻燃剂中, 机理 研究表明, 在燃烧时, 含磷阻燃剂可以同时在气相和固 相中发挥阻燃作用, 且不会产生毒性气体, 是近年来兴 起的一类无卤阻燃剂(图 4). 研究表明, 2-乙基已基二苯 基磷酸酯(EHDPP) 是一种典型的磷酸酯主链结构的聚 合物, 其可作为增塑剂广泛应用于各种产品中, 发挥增 塑和阻燃的双重功能 ${ }^{[8]}$. 除此之外, 含磷低分子聚合 物 ${ }^{[9]}$ 和双酚 $\mathrm{A}$-双 (二苯基磷酸酯)(BDP) ${ }^{[10]}$ 也是商用阻燃 剂的优秀代表.

最后, 磷酸混酯化合物在表面活性剂领域也有一席 之地. 如图 5 所示, 该化合物是一种产率高、性能高效、 易于制备且环保的含氟磷酸酯表面活性剂 ${ }^{[11]}$.

近年来, 随着经济的快速发展, 磷酸混酯类化合物 的需求量逐年增加 ${ }^{[12]}$, 鉴于其在工业和生活中的重要 作用，操作简单、温和高效的合成方法的开发具有非常
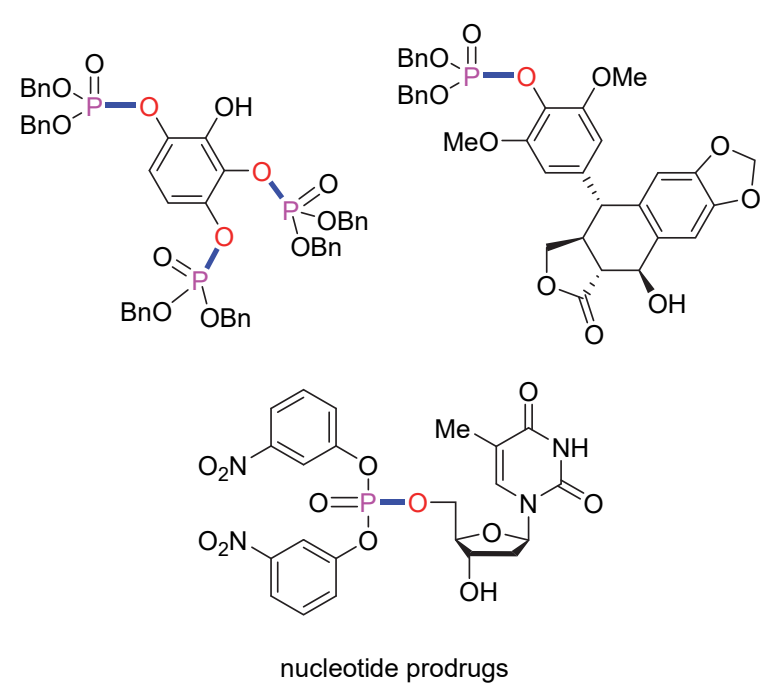

图 3 芳基、烷基磷酸混酯化合物在生物医药方面的应用 Figure 3 Application of aryl and alkyl mixed phosphates in biomedicine

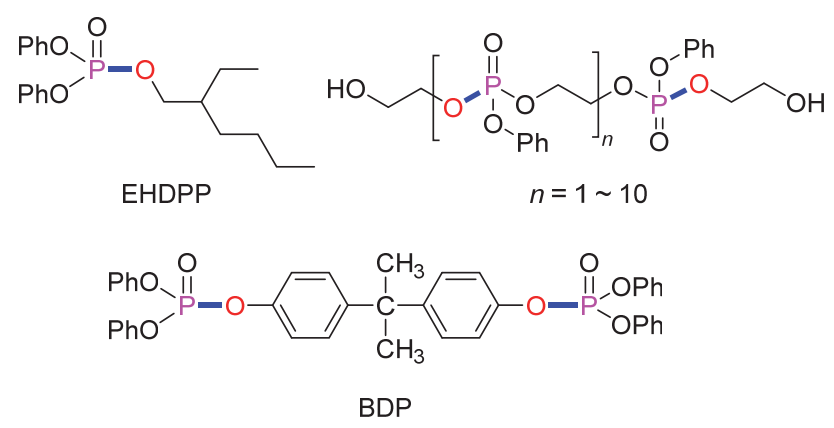

图 4 磷酸混酯化合物在阻燃剂方面的应用

Figure 4 Application of aryl and alkyl mixed phosphates in flame retardant

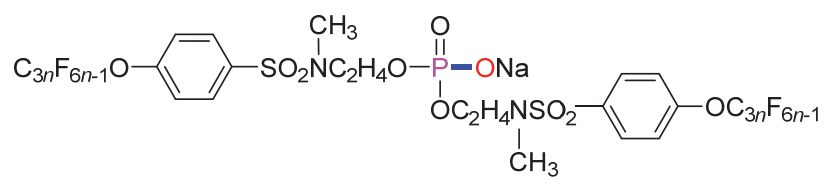

图 5 磷酸混酯类含氟表面活性剂

Figure 5 Mixed phosphates as fluorosurfactant

重要的理论和现实意义. 近年来, 新型、高效的催化手 段和反应体系不断涌现，在一定程度上丰富了起始原料 种类和制备方法，也为科研工作者和工业生产提供了更 多选择. 尽管如此, 截至目前, 尚无磷酸混酯类化合物 制备方法相关的综述报道.

通过系统的文献调研，根据反应底物和反应类型的 不同, 磷酸混酯类化合物的合成方法主要分为以下几 类: 以 $\mathrm{P}(\mathrm{O})-\mathrm{H} 、 \mathrm{P}(\mathrm{O})-\mathrm{Cl} 、 \mathrm{P}(\mathrm{O})-\mathrm{OH} 、 \mathrm{P}(\mathrm{O})-\mathrm{N}_{3}$ 等磷 酰化试剂与亲核试剂(醇、酚等)发生的偶联反应、从三 价磷出发的氧化制备方法、以磷酸酯出发的酯交换法以 及以其它含磷试剂为起始物的合成方法等. 基于以上论 述和事实, 本文将从制备磷酸混酯的不同底物种类出 
发, 系统综述各种磷酰化试剂在向目标化合物转化过程 中的相关研究进展, 有助于读者加深对该领域的了解和 动态掌握, 也希望本文能够起到抛砖引玉的作用, 使更 多的科研工作者参与到相关研究中, 为我国磷化学的发 展贡献力量.

\section{1 基于 $\mathrm{P}(\mathrm{O})$ - $\mathrm{H}$ 键的磷酸混酯化合物的合成 方法}

Atherton-Todd 是合成磷酸酯和膦酸酯的最为经典 的方法之一, 是 $\mathrm{P}-\mathrm{H}$ 键经选择性官能化转化为相应的 $\mathrm{P}-\mathrm{O}$ 和 $\mathrm{P}-\mathrm{N}$ 键的重要途径. 20 世纪 40 年代, Atherton 和 Todd 等 ${ }^{[13]}$ 在非常偶然的情况下, 发现在碱和 $\mathrm{CCl}_{4}$ 的 共同作用下，二烷基膦酸酯可以与胺类化合物反应生成 二烷氧基磷酰胺类化合物，这是目前人们所熟知的 Atherton-Todd 反应的最原始的反应体系(Scheme 1). 两 年之后, Atherton 和 Todd ${ }^{[14]}$ 又进一步对该反应进行了机 理研究, 并将反应底物拓展至水和醇类化合物(Scheme $1)$.

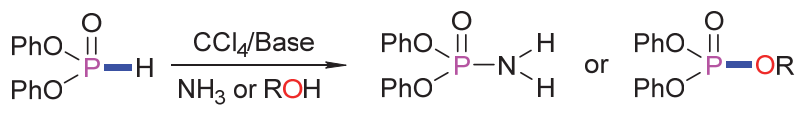

$$
\begin{aligned}
& \text { Yield: } 92 \% \quad \mathrm{R}=\text { alkyl }
\end{aligned}
$$

图式 1 经典 Atherton-Todd 反应通式

Scheme 1 Classical Atherton-Todd reaction formula

近年来，随着合成技术的不断进步，Atherton-Todd 反应的应用范围得到了进一步发展, 不同类型的亲核试 剂均能在相应的催化体系中完成目标转化 ${ }^{[15-16]} .2004$ 年，赵玉芬等通过 Atherton-Todd 反应实现了黄酮类化 合物的磷酸化反应, $\mathrm{CCl}_{4}$ 和 $\mathrm{Et}_{3} \mathrm{~N}$ 的组合试剂为该反应提 供了氯原子, 同时, $\mathrm{Et}_{3} \mathrm{~N}$ 还起到了中和体系中酸性副产 物的作用. 最终, 作者以较高产率得到了黄酮、烷基磷 酸混酯化合物(Scheme 2) ${ }^{[17]}$. 最近, 该课题组又实现了 吡啶氮氧化物邻位甲基的磷酰化反应，其中，氮氧化物 与磷原子进行配位，随后通过 $\mathrm{N} 、 \mathrm{O}$ 以及 $\mathrm{P}$ 原子参与的 $[3,3]-\sigma$ 迁移重排得到磷酸酯化合物(Scheme 3). 值得注 意的是，作者仍然使用 $\mathrm{CCl}_{4}$ 作为氯化试剂，该反应具有 良好的官能团耐受性和较宽的底物范围, 实现了硝酮向 具有潜在生物活性的 $\alpha$-羰基磷酸酯类化合物的高效催 化制备 ${ }^{[18]}$.

从机理角度出发, Atherton-Todd 反应实质上是将 $\mathrm{P}-\mathrm{H}$ 键在体系中原位转化为 $\mathrm{P}-\mathrm{Cl}$ 键, 进而参与反应, 因此, 化学家在新型催化剂/氯化试剂的开发方面取得 了一定进展. 1988 年, Okamoto 课题组 ${ }^{[19]}$ 开发了一种 Atherton-Todd 类型反应, 该反应在催化计量的 $\mathrm{CuCl}_{2}$ 的 作用下，在没有加入氯化试剂 $\mathrm{CCl}_{4}$ 的情况下，顺利实现 了磷酸酯化合物的高效制备, 该反应经历的过渡态如

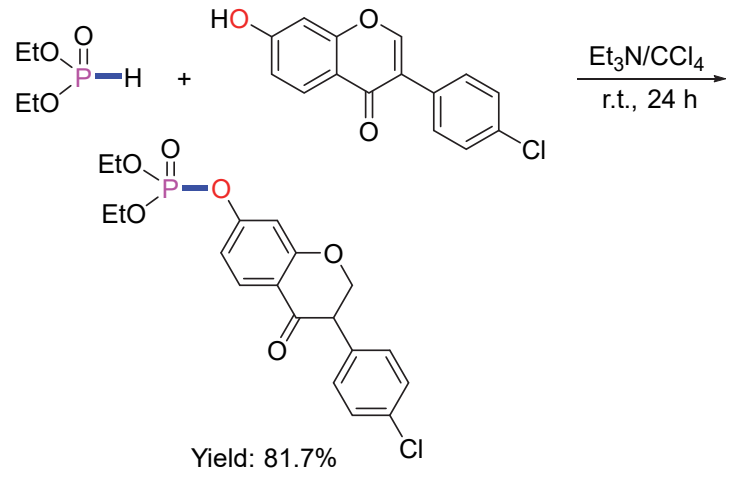

图式 2 黄酮化合物的磷酰化反应

Scheme 2 Phosphorylation of flavonoids

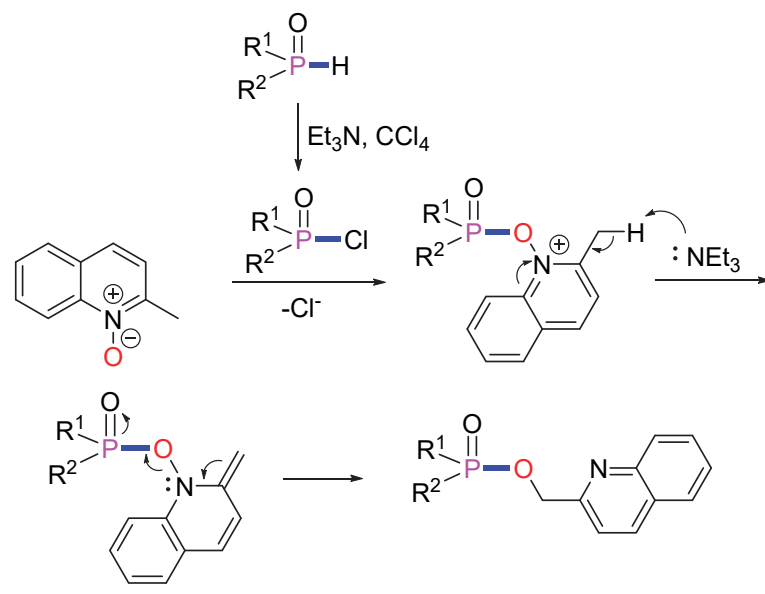

图式 $3[3,3]-\sigma$ 迁移重排制备磷酸混酯

Scheme 3 [3,3]- $\sigma$ migratory rearrangement to prepare mixed phosphates

Scheme 4 所示，首先，P-H 键在催化剂的作用下率先转 化为活性更高的 $\mathrm{P}-\mathrm{Cl}$ 键，同时生成两分子的 $\mathrm{CuCl}$, 然 后经脂肪醇的烷氧基进攻磷酰氯的亲核取代反应得到 目标化合物, 氧气的通入可以将 $\mathrm{Cu}(\mathrm{I})$ 重新氧化为二价 $\mathrm{Cu}(\mathrm{II})$ 参与催化循环.

$$
\begin{aligned}
& \mathrm{PhO}^{\mathrm{PhO}} \stackrel{\mathrm{O}}{\mathrm{P}}-\mathrm{H}+2 \mathrm{CuCl}_{2} \longrightarrow\left[\begin{array}{l}
\mathrm{PhO}_{-}^{\mathrm{O}} \mathrm{P}-\mathrm{Cl} \\
\mathrm{PhO}^{-}
\end{array}\right] \frac{\mathrm{EtOH}}{50^{\circ} \mathrm{C}, 5 \mathrm{~h}} \\
& \text { PhO }{ }_{\text {PhO-P-OEt }} \\
& \text { Yield: 61\% }
\end{aligned}
$$

图式 $4 \mathrm{CuCl}_{2}$ 催化下的 Atherton-Todd 反应制备磷酸酯 Scheme 4 Preparation of phosphate through Atherton-Todd reaction catalyzed by $\mathrm{CuCl}_{2}$

2007 年, Kaushik 等报道了一种在三氯异氧尿酸 (TCICA)存在条件下，通过室温一锅法将二烷基亚磷酸 酯转化为相应的芳基、烷基磷酸酯(Scheme 5). 在该反 应中, TCICA 作为廉价易得、经济绿色的氯化试剂，是 $\mathrm{CCl}_{4}$ 的理想替代品，使得该方法具有简单、高效、环境 友好且易于放大等诸多优点 ${ }^{[20]}$. 最近, Anitha 等报道了 
一种 LiI/TBHP (TBHP: 叔丁基过氧化氢)催化下的 $\mathrm{P}(\mathrm{O})$ $-\mathrm{H}$ 和苯酚、醇、胺等亲核试剂直接磷酰化的方法, 该 催化体系条件温和、快速高效, 且具有良好的官能团耐 受性和较宽的底物应用范围 $(\text { Scheme 6) })^{[21]}$.

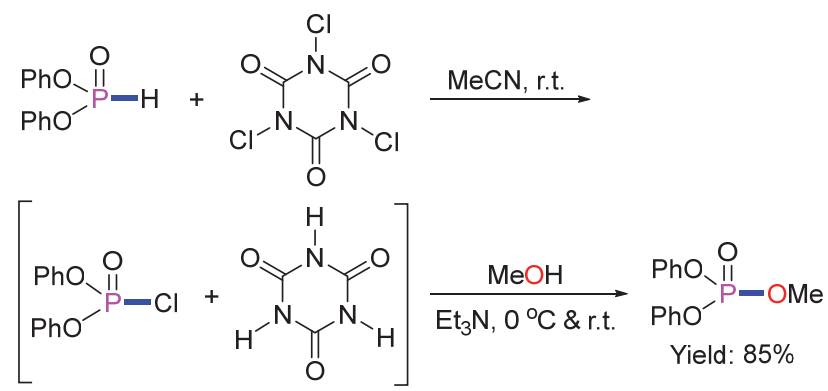

图式 5 TCICA 作用下的一锅法合成磷酸混酯

Scheme 5 One-pot synthesis of mixed phosphate under TCICA<smiles>CCOP(=O)(CC)OCC</smiles><smiles>CCOP(=O)(OCC)Oc1ccc(Br)cc1</smiles>

Yield: $85 \%$

图式 $6 \mathrm{LiI} / \mathrm{TBHP}$ 催化下的芳基、烷基磷酸混酯的合成 Scheme 6 Synthesis of aryl and alkyl mixed phosphates catalyzed by LiI/TBHP

\section{2 基于 $\mathrm{P}(\mathrm{O})-\mathrm{Cl}$ 键的磷酸混酯化合物的合成 方法}

磷酰氯是一类重要的有机磷酰化试剂和合成中间 体, 其中 $\mathrm{Cl}^{-}$具有较高的反应活性和良好的离去性能, 使其在杀虫剂、抗生素、润滑剂、阻燃剂等磷酸酯类精 细化学品的制备中具有重要应用 ${ }^{[22]}$.

Dhawan 等 ${ }^{[23]}$ 在 1986 年报道了一种起始于磷酰氯的 芳基、烷基磷酸酯的制备方法, 作者使用 3 equiv.的吡啶 作为反应促进剂, 在温和 $\left(<40{ }^{\circ} \mathrm{C}\right)$ 条件下顺利实现了 目标转化, 得到 $80 \%$ 的磷酸混酯化合物(Scheme 7), 该 化合物在强碱性(例如二异丙基氨基锂, LDA)条件下可 以发生重排反应, 进而异构化为相应的膦酸酯 $(\mathrm{P}-\mathrm{O}$ 键 异构化为 $\mathrm{P}-\mathrm{C}_{\mathrm{Ar}}$ 键). 需要特别指出的是, 作者在制备 二芳氧基磷酰氯时, 采用三氯氧磷 $\left(\mathrm{POCl}_{3}\right)$ 和苯酚作为 反应物, 在得到磷酰氯的同时, 还生成大量磷酸三芳酯 副产物，因此，底物合成中的选择性是决定该反应成败 问题之一. 无独有偶, 在 2012 年 Page 课题组 ${ }^{[24]}$ 报道的 二苯基磷酰氯和四氢糠醇的酯化反应中, 吡啶同样起着 非常重要的作用(Scheme 8), 它可以在反应过程中中和 生成的酸性 $\mathrm{HCl}$ 副产物, 得到相应的吡啶盐酸盐, 进而 通过体系电导率的变化来表征反应速率.
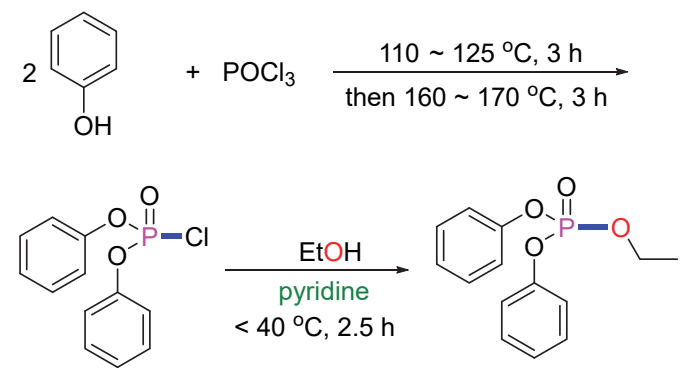

Yield: $80 \%$

图式 7 吡啶促进的磷酰氯和乙醇的酯化反应 Scheme 7 Pyridine promoted esterification of phosphoryl chloride and ethanol

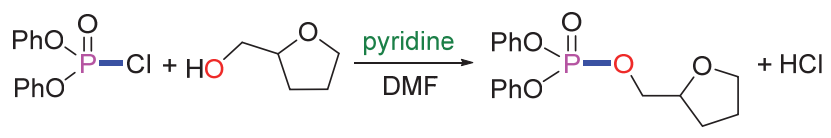

图式 8 吡啶促进的二苯基磷酰氯和四氢糠醇的酯化反应 Scheme 8 Pyridine promoted esterification of diphenylphosphoryl chloride and tetrahydrofurfuryl alcohol

2002 和 2003 年, Jones 等 ${ }^{[25-26]}$ 先后报道了过渡金属 钛作用下磷酰氯的催化转化，证明了 Lewis 酸类催化剂 在芳基、烷基磷酸混酯合成中的重要作用. 研究发现, $\mathrm{TiCl}_{4}$ 和钛酸四叔丁酯都表现出良好的催化性能. 对于 $\mathrm{TiCl}_{4}$ 而言, 有两种可能的反应机理: $\mathrm{Ti}$ 原子既可以先以 Lewis 的形式和磷酰基氧原子配位，降低磷原子的电荷 密度，也可以通过先和脂肪醇发生酯化反应，然后其所 连烷氧基通过分子间的氯和烷氧基迁移互换，得到目标 产物. 而对于 $\mathrm{Ti}$ 中心电荷密度更高的钛酸四叔丁酯，则 更倾向于后者，即先和脂肪醇反应生成三叔丁基烷基钛 酸酯，进而通过分子间的官能团迁移制备相应磷酸酯 (Scheme 9). 不久之后, Gupta 小组 ${ }^{[27]}$ 通过对比 $\mathrm{Al}_{2} \mathrm{O}_{3}$ 存 在与否对脂肪醇膦酰化反应的影响规律, 证实了其良好 的催化性能, 开发了一种简单、高效且环境友好的磷酸 酯制备方法，丰富了 Lewis 酸催化下的转化方式.

2010 年，陈建添等 ${ }^{[28]}$ 报道了一种新型的高效磷酰 化催化剂 $\mathrm{MoOCl}_{4}$, 该催化剂表现出优异的底物兼容性, 在该反应体系中, 对 Lewis 酸较为敏感的糖苷缩醛、乙 酰胺、硅键以及醚键等官能团都可以稳定存在并顺利参 与目标反应. 研究表明, 这种新型的催化体系是一种温 和而实用的磷酸酯合成方法，对于容易发生取代或消除 的不稳定反应底物同样适用; 同时, 具有较大空间位阻 的醇类化合物也表现出较高的反应活性，为该类底物的 磷酰化提供了更多选择. 作者通过 ESI-MS、IR、 ${ }^{1} \mathrm{H}$ NMR 以及 ${ }^{31} \mathrm{P} \mathrm{NMR}$ 等表征方式监测反应进程，找出了涉及的 主要过渡态 $\left[(\mathrm{RO}) \mathrm{Mo}(\mathrm{O}) \mathrm{Cl}_{3}-\mathrm{Et}_{3} \mathrm{~N}\right]$, 并在此基础上提出了 反应的可能机理(Scheme 10). 


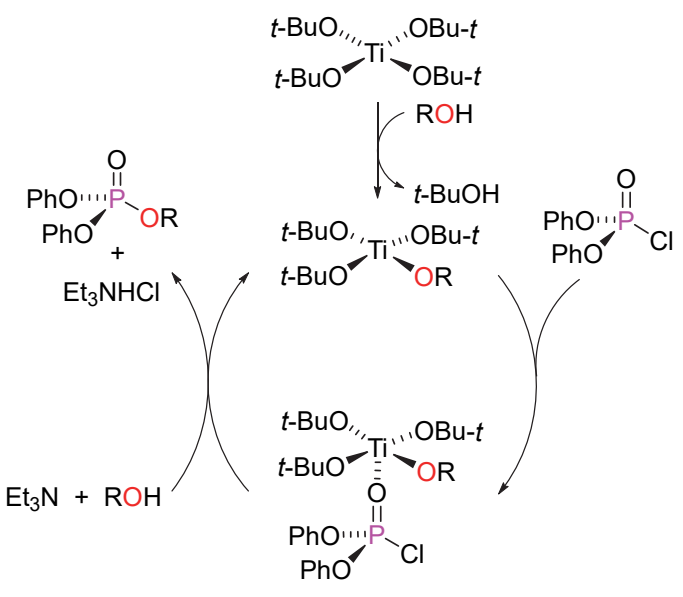

图式 9 钛基 Lewis 酸在磷酸混酯合成中的催化机理 Scheme 9 Catalytic mechanism of titanium-based Lewis acid in the synthesis of mixed phosphates

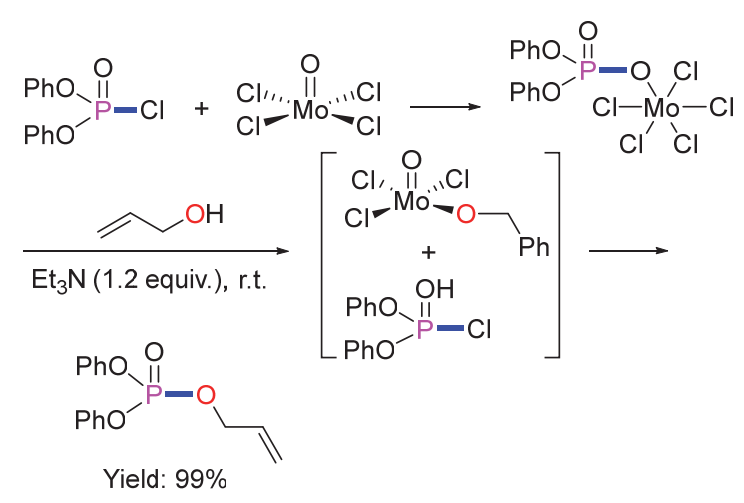

图式 $10 \mathrm{MoOCl}_{4}$ 催化磷酸混酯合成的反应机理

Scheme 10 Reaction mechanism of $\mathrm{MoOCl}_{4}$ catalyzed synthesis of mixed phosphates

张军课题组 ${ }^{[29]}$ 在 2014 年以离子液体 1-烯丙基-3-甲 基咪唑鎓氯化物 $(\mathrm{AmimCl})$ 为反应介质，在均相体系中 合成了一系列新型的含磷纤维素酯, 并首次证了带有短 链和较大位阻基团的含磷官能团对有机物热稳定性的 重要影响, 拓展了离子液体在磷酸酯类化合物合成中的 应用(Scheme 11). 作者通过调节底物的摩尔比以及反应 时间，实现了取代反应进行程度的选择性调控，所得纤 维素酯在常见的有机溶剂中大多具有良好的溶解性，通 过溶液浇铸法制备透明纤维素磷酸酯薄膜. 同时，产物 也具有良好的热塑性, 可以通过传统的熔融方法进行加 工.

最近, Dobrovetsky 和 Portno 等[30]将脂肪醇和磷酰氯 的催化剂种类拓展至有机小分子催化体系，作者合成了 咪唑类和二甲基氨基吡啶类两个系列的催化剂, 并将其 应用于目标转化中. 相对而言, 带有三聚乙二醇链取代 基的催化剂表现出最佳的催化性能, 无法通过常规的电 子效应和空间位阻效应进行解释(Scheme 12). 通过基 于实验设计和密度泛函理论计算(DFT)的机理研究, 作
者最终证明，咪唑或吡啶与磷酰化试剂形成了 $\mathrm{P}-\mathrm{N}$ 类 氮杂环阳离子中间体，而催化过渡态中氧原子的孤对电 子对其的配位作用，是使后者形成稳定中间体的关键步 骤(Scheme 12).

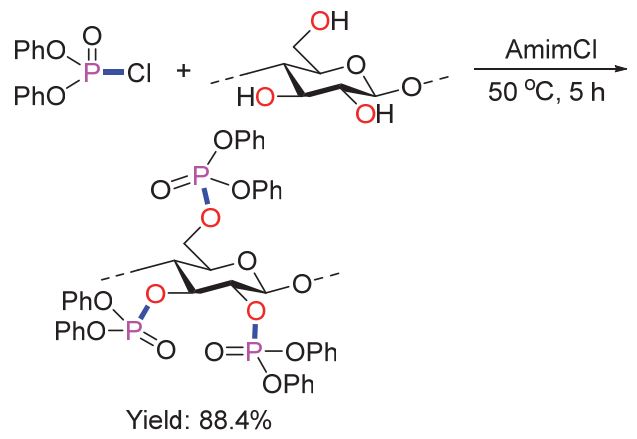

图式 11 磷酸纤维素二苯酯在离子液体中的合成 Scheme 11 Synthesis of cellulose diphenyl phosphate in ionic liquid

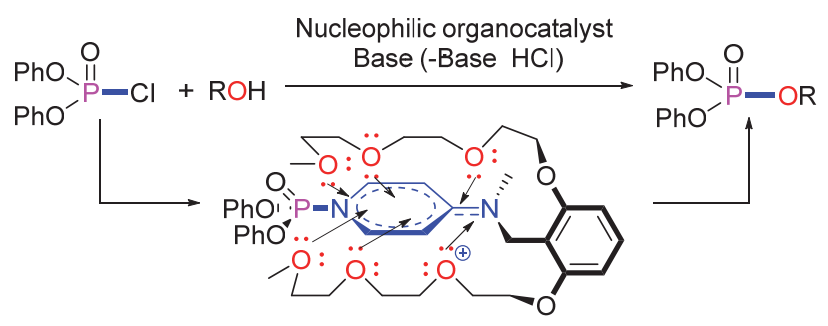

$\mathrm{R}=$ alkyl, Yield: $21 \% \sim 77 \%$

图式 12 基于聚乙二醇链的催化剂在合成磷酸混酯中的应用 Scheme 12 Application of catalysts based on polyethylene glycol chains in the synthesis of mixed phosphates

\section{3 基于 $\mathrm{P}(\mathrm{O})-\mathrm{OH}$ 键的磷酸混酯化合物的合成 方法}

2003 年, Bräse 课题组 ${ }^{[31]}$ 开发了一种新型的磷酸酯 制备方法，作者从磷酸二苯酯出发，和基于聚合物的烷 基三氮烯选择性发生偶联反应，以 $59 \% \sim 65 \%$ 的产率制 备了芳基、烷基磷酸混酯(Scheme 13). 在该体系中, 无 需任何催化剂，三氮烯上所连烷基特别是甲基表现出非 常高的反应活性，为目标化合物的制备提供了新的途 径.

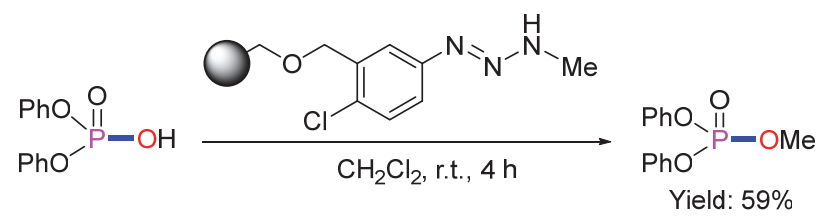

图式 13 与聚合物结合的三氮烯催化 $\mathrm{P}(\mathrm{O})-\mathrm{OH}$ 合成磷酸混 酯类化合物

Scheme 13 Triazene combined with polymer catalyzed $\mathrm{P}(\mathrm{O})$ $\mathrm{OH}$ synthesis of mixed phosphatess 
2006 年和 2007 年, Kaushik 等先后从磷酸二苯酯制 备磷酸混酯的研究中发现, 氯化硅 $\left(\mathrm{SiO}_{2}-\mathrm{Cl}\right)$ (Scheme $14)^{[32]}$ 和对甲苯磺酸-硅藻土复合材料 $(p \text {-TsOH-Celite })^{[33]}$ 均表现出较高的非均相催化活性, 特别是后者, 两种反 应底物磷酸二苯酯和乙醇的物质的量比仅为 $1: 1$, 反 应可以顺利进行(Scheme 15), 上述两种方法操作简单、 条件温和且转化效率高, 在室温 $\sim 40{ }^{\circ} \mathrm{C}$ 条件下反应 10 40 min, 即可高效得到目标产物, 分离产率高达 $96 \%$.

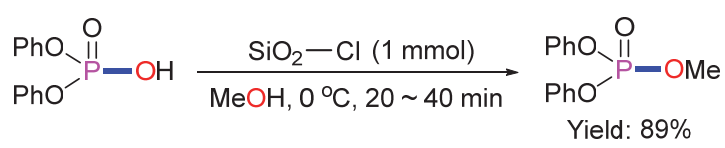

图式 $14 \mathrm{SiO}_{2}-\mathrm{Cl}$ 催化的以 $\mathrm{P}(\mathrm{O})-\mathrm{OH}$ 化合物的酯化反应 Scheme 14 Esterification of $\mathrm{P}(\mathrm{O})-\mathrm{OH}$ compound catalyzed by $\mathrm{SiO}_{2}-\mathrm{Cl}$

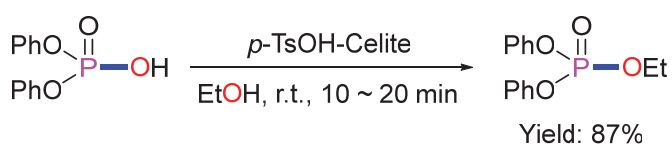

Conditions: (1) without solvent; (2) $(\mathrm{PhO})_{2} \mathrm{P}(\mathrm{O}) \mathrm{OH}$ : p-TsOH:Celite: $\mathrm{EtOH}=1: 1: 1: 1$

图式 $15 p$-TsOH-Celite 催化的以 $\mathrm{P}(\mathrm{O})-\mathrm{OH}$ 化合物的酯化反 应

Scheme $15 p$-TsOH-celite catalyzed esterification reaction with $\mathrm{P}(\mathrm{O})-\mathrm{OH}$ compound

2011 年, Chavasiri 和 Jang 等 ${ }^{[34]}$ 开发了一种简单、便 捷的 “一锅法” 来合成磷酸酯类化合物. 该方法反应条 件温和, 反应时间短且易于操作. 作者以 $\mathrm{CH}_{2} \mathrm{Cl}_{2}$ 作为溶 剂, 以三乙胺 $\left(\mathrm{Et}_{3} \mathrm{~N}\right)$ 或者吡啶等含氮化合物作为碱性添 加剂, 在三苯基膦 $\left(\mathrm{PPh}_{3}\right)$ 和三氯乙腈 $\left(\mathrm{CCl}_{3} \mathrm{CN}\right)$ 的共同作 用下，实现了磷酸二苯酯与醇类化合物的酯化反应 (Scheme 16). 研究表明, 当反应体系中 $\mathrm{Ph}_{3} \mathrm{P}$ 用量为 2 equiv. $\mathrm{Cl}_{3} \mathrm{CCN}$ 用量为 3 equiv.时, 酯化反应的效率最高, 达到 $92 \%$.

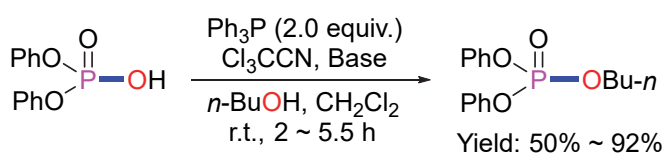

图式 $16 \mathrm{PPh}_{3}$ 和三氯乙腈作用下经 “一锅法” 合成磷酸混酯 Scheme 16 Synthesis of mixed phosphates by "one-pot reaction" with the promotion of $\mathrm{PPh}_{3}$ and trichloroacetonitrile

2014 年, 尹双凤等 ${ }^{[35]}$ 开发了一种通过过量碱促进 的二苯基磷酸与卤代烷烃化合物(如茮澳)合成磷酸酯的 新方法(Scheme 17). 该体系没有使用任何对空气敏感 的化学试剂, 很容易地将廉价易得的 $\mathrm{P}(\mathrm{O})-\mathrm{OH}$ 化合物 转化为各种功能性取代的有机磷化合物, 高效地制备了 一系列芳基、烷基磷酸混酯和烷基、烷基磷酸混酯, 在
生物活性分子、催化配体和有机磷材料的构建中具有潜 在的应用价值.

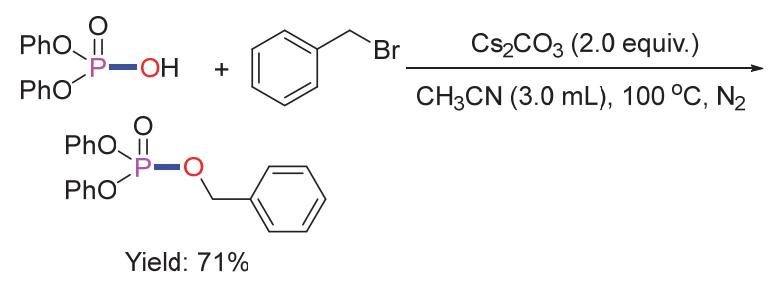

图式 17 碱促进的二苯基磷酸与苄溴反应制备磷酸混酯 Scheme 17 Alkali-promoted reaction of diphenylphosphoric acid with benzyl bromide to prepare mixed phosphates

鉴于过渡金属催化剂在化学键构筑领域表现出的 较高催化性能, 2015 年, 该课题组 ${ }^{[36] 又}$ 开发了一种铜催 化的 $\mathrm{P}(\mathrm{O})-\mathrm{OH}$ 化合物和醇的酯化反应，均得到较好的 催化结果，以中等到较高的分离产率制备了一系列磷酸 酯、膦酸酯以及次膦酸酯(Scheme 18, Path A). 进一步研 究发现，当使用二芳基碘鉾盐代替醇作为偶联试剂时， 作者成功地将该转化拓展至芳基酯化试剂. 然而，值得 注意的是, 该反应无需添加 $\mathrm{Cu}$ 催化剂, 在 $110{ }^{\circ} \mathrm{C}$ 和三 乙胺辅助条件下反应即可顺利进行, 而且表现出较高的 底物适用性和官能团耐受性(Scheme 18, Path B) ${ }^{[36]}$.

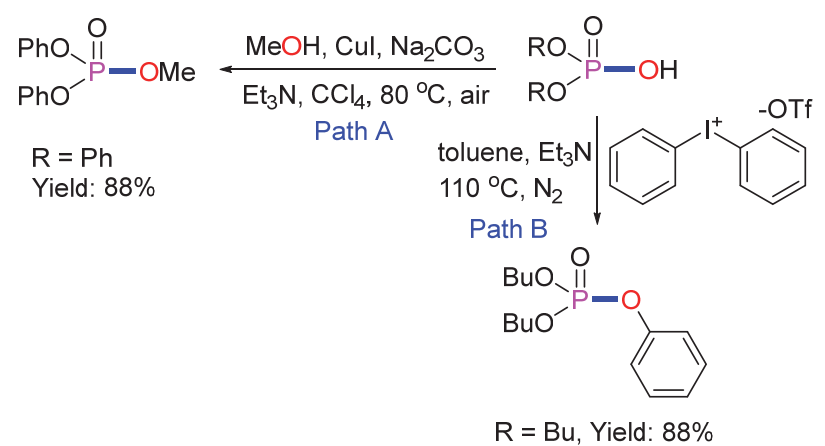

图式 $18 \mathrm{P}(\mathrm{O})-\mathrm{OH}$ 和脂肪醇以及二芳基碘鎓盐的酯化反应 Scheme 18 Esterification of $\mathrm{P}(\mathrm{O})-\mathrm{OH}$ with aliphatic alcohols and diaryliodonium salts

近年来，熊碧泉和唐课文课题组先后报道了将 $\mathrm{Cu}$ 催化的 Chan-Lam 类型的偶联反应 ${ }^{[37]} 、 N, N^{\prime}$-羰基二咪唑

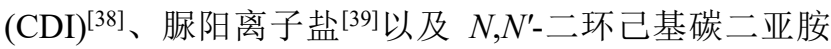
(DCC) ${ }^{[40]}$ 催化的酯化反应，应用于芳基、烷基磷酸混酯 的制备中, 然而, 反应活性较低, 结果也不尽如人意.

\section{4 基于三价磷的磷酸混酯化合物的催化合成方 法}

在有机磷化合物中，磷原子的价态具有多样性. 其 中, 亚磷酸及其衍生物是非常常见且重要的三价磷化合 物，通过定向氧化将其转化为五价磷也是制备磷酸酯的 有效途径之一。 
早在 1983 年, Bartlett 等就报道了一种亚磷酸酯经 臭氧 $\left(\mathrm{O}_{3}\right)$ 氧化、分解等步骤制备芳基、烷基磷酸酯的方 法 ${ }^{[41]}$. 如 Scheme 19 所示, 作者以亚磷酸三苯酯为底物, 在 $-78{ }^{\circ} \mathrm{C}$ 的臭氧体系中反应 $7 \mathrm{~d}$, 得到一种 $\mathrm{P}-\mathrm{O}-\mathrm{O}-$ $\mathrm{O}$ 首尾相连的四元环臭氧化物中间体, 该化合物非常不 稳定, 其活性在 $-78{ }^{\circ} \mathrm{C}$ 的极低温度条件下, 仅能保持 6 $\mathrm{h}$. 接着, 在吡啶的作用下, 甲氧基取代苯氧基得到二苯 基甲基磷过渡态. 由于芳氧基和烷氧基更倾向于处于磷 三角双雉形状的赤道平面, 因此臭氧化物很容易经 Stephenson-McClure 类型的分解反应, 磷和臭氧组成的 四元环断裂, 三个氧原子分别位于三角双雉的顶部和底 部位置, 该化合物可以进一步分解释放出单线态分子氧 $\left({ }^{1} \mathrm{O}_{2}\right)$ 的同时, 得到甲基二苯基磷酸酯 ${ }^{[42]}$. 虽然该方法存 在条件苛刻、难以有效控制等明显的局限性，但目前仍 然是最早的磷酸混酯的氧化制备途径.

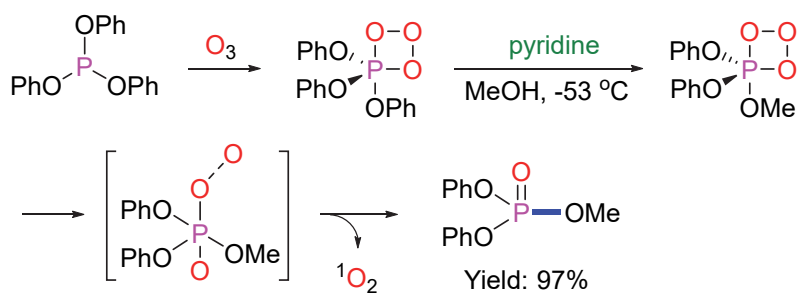

图式 19 亚磷酸酯经氧化和分解制备磷酸混酯

Scheme 19 Preparation of mixed phosphates by oxidation and decomposition of phosphite

2009 年, Oba 课题组 ${ }^{[43]}$ 在这一领域取得重大突破, 报道了一种光催化条件下的高效氧化(Scheme 20). 作 者以孟加拉玫瑰红(rose bengal)作为光敏化剂, 在光照 条件下诱导三线态分子氧 $\left({ }^{3} \mathrm{O}_{2}\right)$ 转化为单线态分子氧 $\left({ }^{1} \mathrm{O}_{2}\right)$, 后者具有相对较低的活化能，可以实现二芳基碲 化物 $\left(\mathrm{Ar}_{2} \mathrm{TeO}\right)$ 的催化氧化. 在亚磷酸酯存在的条件下, 碲氧化物经氧原子转移，顺利实现五价的二苯基甲基磷 酸混酯的高效催化制备, 分离产率达 $91 \%$. 该催化氧化 效果非常好, 并且对环境友好; 值得注意的是, 在特定 情况下，有机碲催化剂甚至可以定量回收和再利用.

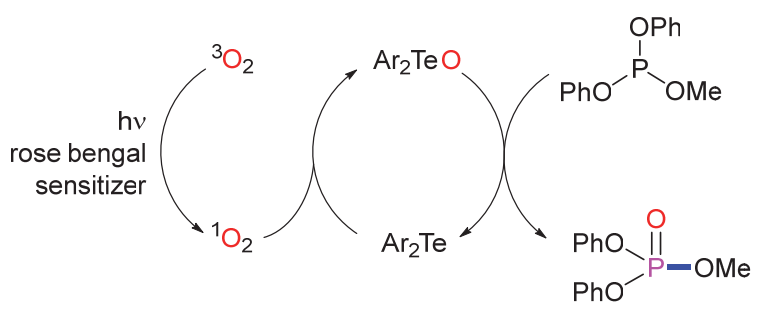

Yield: $91 \%$

图式 20 光诱导的芳基、烷基磷酸混酯的催化制备 Scheme 20 Catalytic preparation of light-induced aryl and alkyl mixed phosphates

\section{5 基于酯交换的磷酸混酯化合物的合成方法}

磷酸酯在有机化工和农业化工领域广泛用作杀虫 剂和农药，人们在充分利用其良好生物活性的同时，作 为神经毒剂, 农药的过量使用会对人体和环境造成很大 的危害，因此，该类化合物的有效降解和污染区域的化 学修复势在必行.

2010 年, Tamilselvi 和 Mugesh 等[44]设计合成了一系 列单中心和双中心的 $\mathrm{Zn}$ 配合物，并将其应用于磷酸三 芳基酯(PNPDPP)在 $\mathrm{pH}$ 为 9.0 的 2-环己胺基乙磺酸 (CHES)缓冲液中的水解反应, 然而效果不佳. 经过进一 步研究发现，当使用甲醇作为替代溶液时，底物的醇解 速率远高于其在缓冲溶液中的水解速率，甲氧基对磷中 心原子的进攻使得相对较弱的 $\mathrm{P}-\mathrm{O}$ 键断裂，高产率、 高选择性地得到低毒的二苯基甲基磷酸酯, 并得到一分 子对硝基苯酚(PNP), 实现了污染物的高效降解(Scheme 21). 在该反应中，底物的半衰期约为 $202 \mathrm{~min}$.

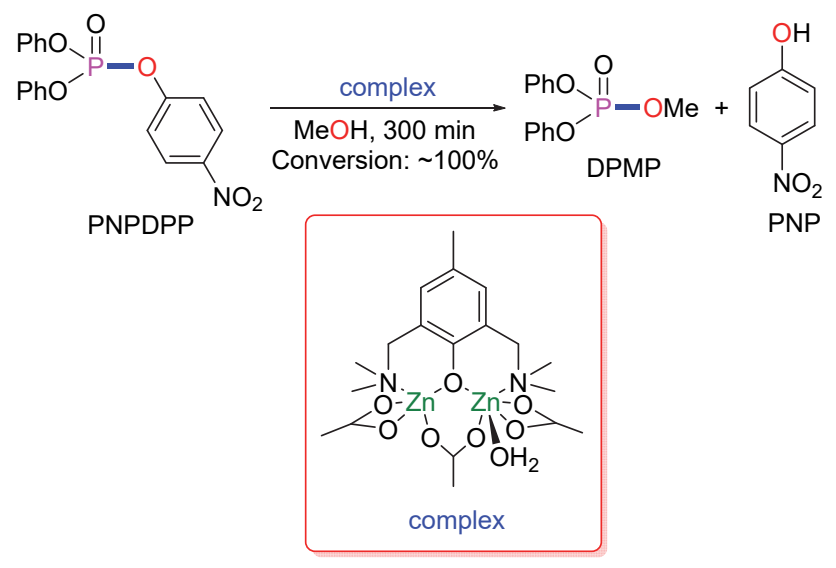

图式 21 磷酸三芳基酯在锌配合物作用下的醇解反应 Scheme 21 Alcoholysis of triaryl phosphate under the action of zinc complex

近年来，金属卟啉以其良好的光、电化学性能，在 有机污染物的降解方面具有广泛的应用. 2012 年, Hupp 和 Nguyen 等 ${ }^{[45-46]}$ 先后报道了锌/铝卟啉在磷酸酯的酯交 换反应中的催化活性. 研究表明，无论是二聚体的金属 卟啉(如铝卟啉, Scheme 22), 还是盒状的金属卟啉四聚 体超分子体系，都可以高效催化二苯基对硝基苯基磷酸 的选择性醇解反应，得到相应的酯交换产物二苯基甲基 磷酸酯. 然而, 该反应的活性较低, 原料经过 $54 \mathrm{~h}$ 的转 化，只得到 $74 \%$ 的醇解产物.

金属有机框架(MOF) 是近年来兴起并快速发展的 一种新型材料和高性能催化剂. 在上述研究的基础上, 两年之后，该课题组又将醇解的催化体系拓展至 $\mathrm{MOF}$ 材料, 其中, UIO-66 以加强 Lewis 酸性中心 $\mathrm{Zr}^{\mathrm{IV}}$ 以及氢 
氧化物阴离子桥键的作用, 待降解物质的半衰期约为 45 50 min, 只有之前报道 ${ }^{[44]}$ 的四分之一左右. 因此, 在该条件下, 底物水解和醇解的转化效率都得到有效提 高(Scheme 23) ${ }^{[47]}$.

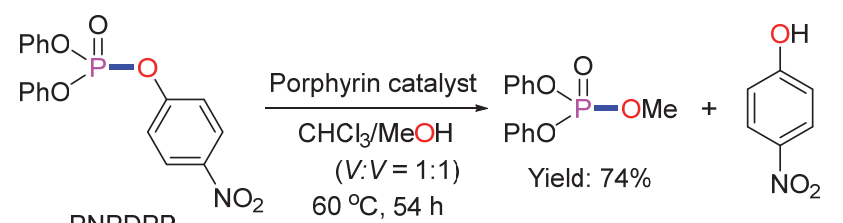
PNPDPP

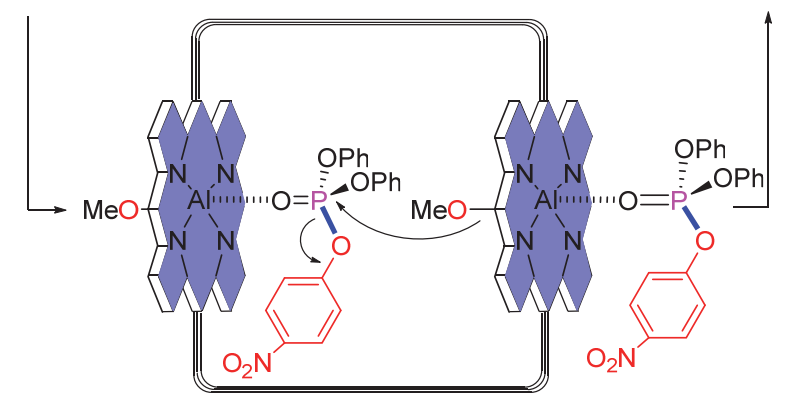

图式 22 铝卟啉二聚体催化的磷酸三芳基酯的醇解反应 Scheme 22 Alcoholysis of triaryl phosphate catalyzed by aluminum porphyrin dimer

(a) Hydrolysis

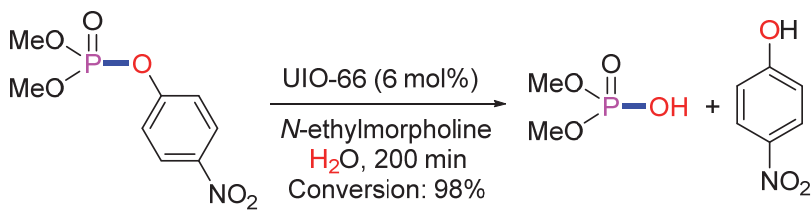

(b) Alcoholysis

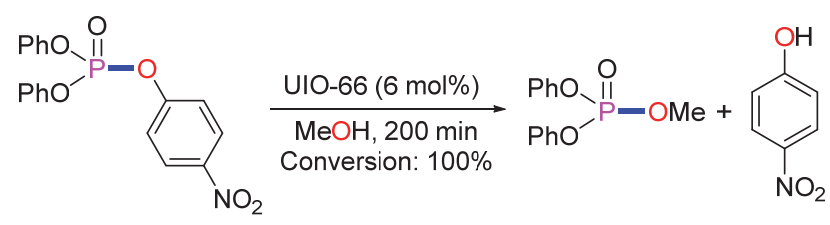

图式 23 UIO-66 催化的磷酸三芳基酯的水解和醇解反应

Scheme 23 Hydrolysis and alcoholysis of triaryl phosphate catalyzed by UIO-66

2018 年, 康俊勇课题组 ${ }^{[48]}$ 开发了一种温和、直接的 通过 $\mathrm{Tf}_{2} \mathrm{O}$ /吡啶催化体系促进的酯交换反应(Scheme 24), 与上述酯交换或者醇解反应不同, 该转化可以将磷酸酯 中的烷氧基选择性地转化为芳氧基, 得到芳基、烷基磷 酸酯. 研究表明, 该体系无需使用金属或氯化物试剂即 可合成多种功能性混合磷酸酯类化合物, 转化过程中生 成的亲电型磷物种可以与多种亲核试剂(如脂族醇、苯 酚、胺以及硫醇等)发生亲核取代反应, 作者还将催化反 应拓展至连续流动反应体系(Continuous Flow System), 实现了目标化合物的克级制备. 反应机理表明, 吡啶在 转化过程中的作用至关重要, 可以和磷中心形成磷酸酯 吡啶盐中间体 ${ }^{[49]}$, 为后续芳氧基的亲核取代提供可能.

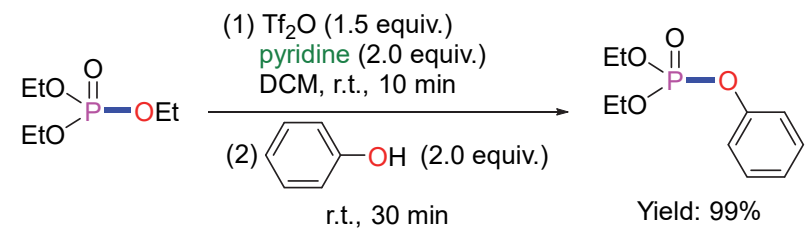
(1) $\mathrm{Tf}_{2} \mathrm{O}$ (1.5 equiv.) pyridine (2.0 equiv.) DCM, r.t., $10 \mathrm{~min}$
(2) $\mathrm{Me} \longrightarrow \mathrm{OH}$ (2.0 equiv.) r.t., $30 \mathrm{~min}$

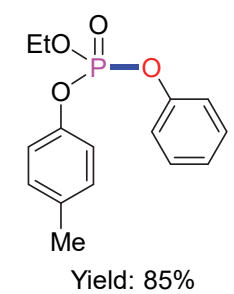

Yield: $85 \%$
图式 $24 \mathrm{Tf}_{2} \mathrm{O}$ 吡啶促进的连续酯交换反应 Scheme $24 \quad \mathrm{Tf}_{2} \mathrm{O} /$ pyridine promoted continuous transesterification reaction

在该类转化中, 酸性氧化物也可以起到较好的促进 作用. 2001 年, Kaboudin 等 ${ }^{[50]}$ 开发了一种用磷酸三乙酯/ 五氧化二磷 $\left(\mathrm{P}_{2} \mathrm{O}_{5}\right)$ 的混合物处理酚类化合物的方法, 在 $120{ }^{\circ} \mathrm{C}$ 条件下, 有机磷试剂和取代苯酚经过 $48 \mathrm{~h}$ 反应, 可以以中等到较高产率得到单酯交换产物.

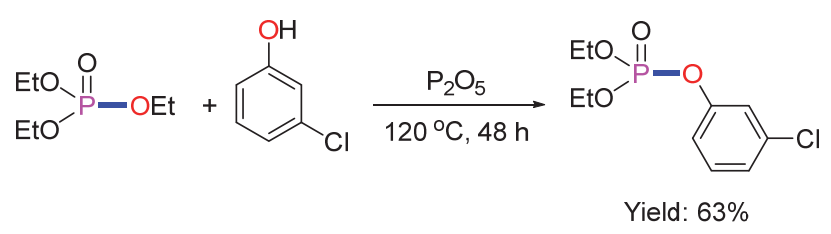

图式 $25 \mathrm{P}_{2} \mathrm{O}_{5}$ 促进的磷酸三乙酯的酯交换反应

Scheme 25 Transesterification of triethyl phosphate promoted by $\mathrm{P}_{2} \mathrm{O}_{5}$

\section{6 基于磷酰叠氮的磷酸混酯化合物的合成方法}

通过前面的论述可知, 合成磷酸酯类化合物通常是 以 $\mathrm{P}(\mathrm{O})-\mathrm{H} 、 \mathrm{P}(\mathrm{O})-\mathrm{Cl} 、 \mathrm{P}(\mathrm{O})-\mathrm{OH}$ 官能团作为原料, 以 醇、酚、胺以及硫醇等作为亲核试剂发生反应, 通常需 要使用有毒或者稳定性较差的反应底物或者试剂、催化 剂等, 会对设备造成腐蚀和破坏, 此外, 有些反应需要 在较高温度或者无水无氧的条件下才能顺利进行, 在一 定程度上限制了反应的普适性和放大操作. 因此, 开发 新的高效的合成磷酸混酯类化合物的工艺迫在眉睫.

叠氮磷酸二苯酯(DPPA) 是一种多功能的有机磷试 剂, 截至目前, 已经在叠氮化反应 ${ }^{[51]}$ 、过渡金属催化的 胺基化反应 ${ }^{[52] 、}$ 改进 Curtius 重排反应 ${ }^{[53]}$ 以及选择性诱 导酰胺键形成 ${ }^{[54]}$ 等诸多转化中表现出良好的反应活性 和产物选择性.

2019 年, 焦林郁等 ${ }^{[7]}$ 首次将 DPPA 作为磷酰化试剂 应用于芳基、烷基磷酸混酯的催化合成中(Scheme 26). 在该催化体系中, DPPA 表现出不同于已知的过渡金属 或者强碱条件下的断键方式, $\mathrm{CuCl}$ 的催化作用不但使得 
$\mathrm{P}-\mathrm{N}_{\alpha}$ 键发生选择性断裂, 在离去一分子 $\mathrm{N}_{2}$ 的同时, 得 到 $\mathrm{N}=\mathrm{Cu}$ 金属乃春中间体, 而且还可以以 Lewis 的形式 和 $\mathrm{P}=\mathrm{O}$ 双键中的氧原子配位, 从而有效降低磷原子的 中心电荷密度, 促进烷氧基亲核取代反应的进行.

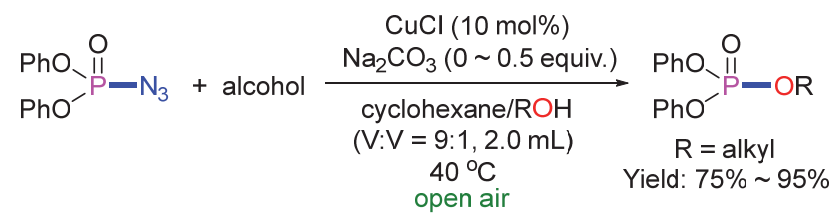

图式 26 叠氮磷酸二苯酯和脂肪醇在铜作用下的催化转化 Scheme 26 Catalytic conversion of diphenyl azide phosphate and fatty alcohol under the action of copper

随后, 吴小锋课题组等 ${ }^{[55]}$ 实现了 $\mathrm{Zn}(\mathrm{acac})_{2}$ 在温和 条件下的选择性催化转化, 在该体系中, 脂肪醇和胺均 可顺利地与 DPPA 反应, 高效合成了一系列二苯基磷酸 酯(Scheme 27)和磷酰胺化合物. 具有相同碳原子的脂 肪醇和脂肪胺的分子间竞争反应显示, 胺类的亲核性较 醇类略高, 得到两种产物的混合体系(磷酸酯: 磷酰 胺 $=52: 48)$.

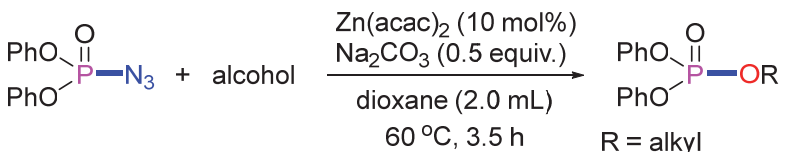

$$
\begin{aligned}
& \text { Yield: 43\% 90\% }
\end{aligned}
$$

图式 27 叠氮磷酸二苯酯和脂肪醇在锌作用下的催化转化 Scheme 27 Catalytic conversion of diphenyl azide phosphate and aliphatic alcohol under the action of zinc

最近，焦林郁等 ${ }^{[56] 又}$ 将过渡金属催化的基于 DPPA 的磷酸混酯合成方法拓展至多相体系, 作者选用壳聚糖 $(\mathrm{CS})$ 作为天然多相负载剂, 通过简单合成制备了负载型 铜催化剂. 虽然其反应活性较均相体系相比略有降低, 但是仍然可以在空气中实现 DPPA 向酯化产物的高效转 化，以中等至优异的分离产率合成了多种芳基、烷基磷 酸酯类化合物, 为目标化合物的合成提供了更多选择 (Scheme 28). 研究表明, 反应结束之后, 负载型催化剂 可以通过简单过滤实现回收和再利用, 具有一定的循环
稳定性. 通过对比，反应前后催化剂的形貌、结构以及 中心金属离子的价态均没有明显变化，随着催化剂使用 次数的增加, 其中的铜含量逐渐降低, 表明活性铜物种 在反应进程或后处理过程中被少量浸出, 这是造成催化 活性降低的主要原因.

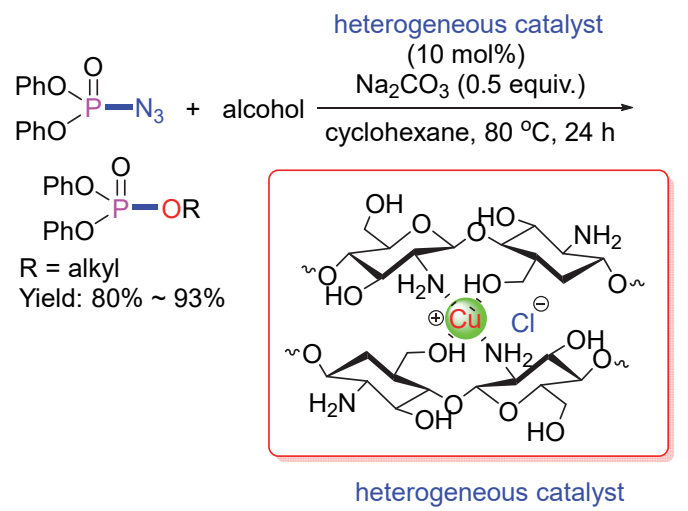

图式 28 叠氮磷酸二苯酯在多相体系下的催化转化 Scheme 28 Catalytic conversion of diphenyl azide phosphate in heterogeneous system

\section{7 基于其它磷酰化试剂的磷酸混酯化合物的合 成方法}

除了上述磷酰化试剂和方法，当含磷底物分子中连 有吡啶、氧基、三唑、噻唑以及噁唑烷酮等离子或官能 团时，也可以作为芳基、烷基磷酸混酯前体，在一定条 件下转化为目标产物.

20 世纪 70 年代, Mitsunobu 等[57]通过二芳氧基磷酰 氯和 1,4-二氢吡啶的化学反应制备了磷酰胺 (Scheme 29)，该化合物经还原型辅酶 I (Nicotinamide adenine dinucleotide, NADH)简单作用得到磷酰基的吡啶鎓盐中 间体，后者分别在三苯基碳正离子(乙腈中回流反应)或 者 $\mathrm{Ce}^{\mathrm{IV}}$ 离子(脂肪醇中室温反应)的作用下与脂肪醇发生 亲核取代制备芳基、烷基磷酸混酯(Scheme 29).

接着, 基于 1,4-二氢吡啶在光照条件下潜在的衍生 化性能，作者又将上述反应应用于高压录灯照射下的以

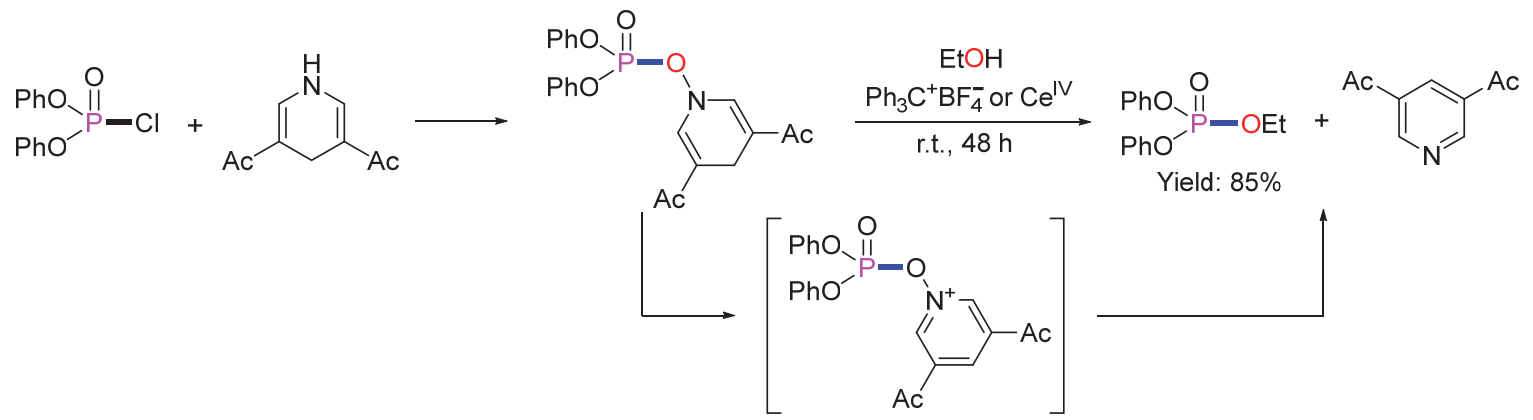

图式 29 磷酰吡啶鎓盐中间体在磷酸混酯合成中的应用

Scheme 29 Application of phosphorylpyridinium salt intermediate in the synthesis of mixed phosphates 
$\mathrm{O}_{2}$ 为氧化剂的反应体系中, 通过光氧化反应一步制备 了磷酸酯化合物, 反应速率得到大幅提高 (Scheme $30)^{[57]}$.

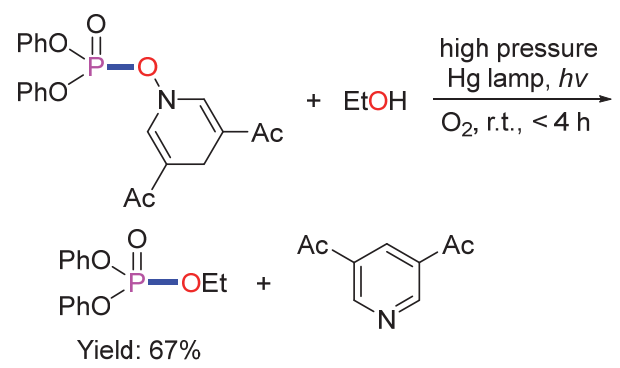

图式 30 高压录灯条件下磷酰吡啶鎓盐在磷酸混酯合成中的 应用

Scheme 30 Application of phosphorylpyridinium salt in the synthesis of mixed phosphates under the condition of high pressure mercury lamp

2002 年, Tanaka 课题组 ${ }^{[58]}$ 开发了一种新型的磷酰化 试剂, 并将其成果应用于和脂肪醇的反应中, 高效制备 芳基、烷基磷酸混酯类化合物(Scheme 31). 作者选用氧 基亚氨基噻唑烷(NCT)作为高效的胺基化试剂, 在常温 下和磷酰氯在 $\mathrm{Et}_{3} \mathrm{~N}$ 和 $\mathrm{CH}_{2} \mathrm{Cl}_{2}$ 溶液中经一步反应制备磷 酰胺化合物(Scheme 32). 当与脂肪醇进行亲核反应时, NCT 很容易因被取代而离去, 合成目标产物的同时, 也 得到了一分子的噻唑烷副产物, 因此, 该反应本质上也 属于磷酰氯和脂肪醇在仲胺参与下的酯化反应. 此外, 研究证明, 叔丁基格氏试剂的存在对目标反应至关重 要, 使得反应可以在温和条件下快速转化.

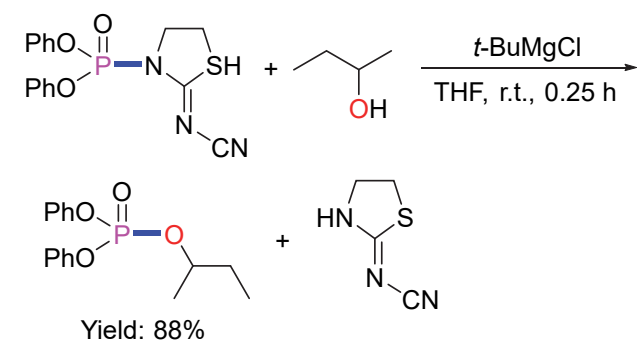

图式 31 基于 NCT 的新型磷酰化试剂在磷酸混酯合成中的应 用

Scheme 31 Application of NCT-based new phosphorylation reagents in the synthesis of mixed phosphates

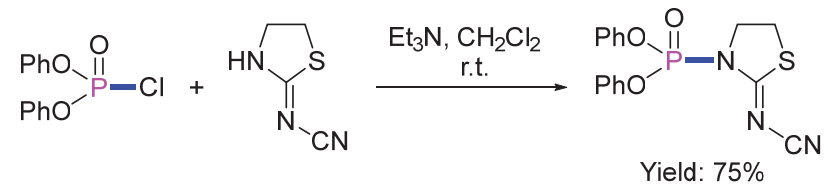

图式 $32 \mathrm{NCT}$ 与磷酰氯制备磷酰胺化合物

Scheme 32 Preparation of phosphoramide compounds from NCT and phosphoryl chloride

两年以后, Jones 等 ${ }^{[59]}$ 将啞唑烷酮与正丁基锂
( $n$ - $\mathrm{BuLi}) 、 \mathrm{THF}$ 和磷酰氯反应, 设计合成了一种新型磷 酰化试剂(Scheme 33)。研究表明, 在有机金属试剂 $n$-BuLi 的作用下, 磷酰化试剂可以和脂肪醇进行酯化得 到芳基、烷基磷酸酯，噁唑烷酮中羰基氧原子和磷酰基 氧原子都可以和锂离子进行配位，形成六元环中间体， 噁唑烷酮中碳原子的取代基的大小和种类对目标反应 具有较大影响(Scheme 34). 值得注意的是, 等物质的量 的活泼有机锂试剂的使用，也使得该转化局限于绝对无 水无氧反应体系, 对反应条件和操作技术具有较高的要 求.

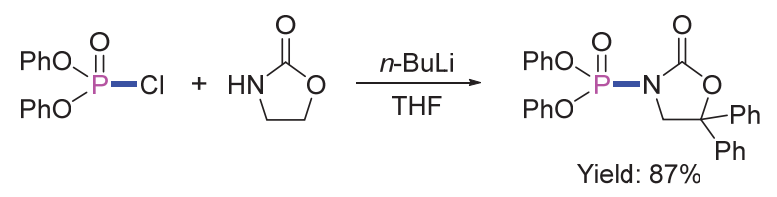

图式 33 噁唑烷酮与磷酰氯合成新型磷酰化试剂

Scheme 33 Synthesis of novel phosphorylation reagents from oxazolidinone and phosphoryl chloride

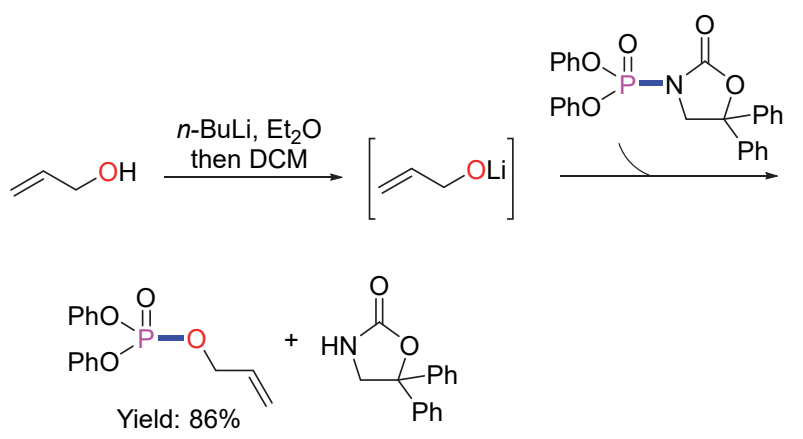

图式 34 含有噁唑烷酮的新型磷酰化试剂在磷酸酯合成中的 应用

Scheme 34 Application of new phosphorylation reagents containing oxazolidinone in the synthesis of phosphates

不久之后，该课题组 ${ }^{[60]}$ 又实现了上述反应体系在 $\mathrm{Cu}(\mathrm{OTf})_{2}$ 和含氮配体 $N, N^{\prime}$-双(苯基亚甲基)-1,2-乙二胺 (BEN)的共同作用下的催化转化, 使得反应条件较之前 变得更为简单温和(Scheme 35). 在该催化体系中, 伯 醇、仲醇、叔醇以及苠醇等脂肪醇均能高效转化为相应 的芳基、烷基磷酸混酯化合物. 除了铜系催化剂，碱金 属盐 $\mathrm{Mg}(\mathrm{OTf})_{2}$ ，稀土金属三氟甲磺酸盐 $\mathrm{Gd}(\mathrm{OTf})_{3}$ 、 $\mathrm{Y}(\mathrm{OTf})_{3}$ 以及 $\mathrm{Eu}(\mathrm{OTf})_{3}$ 也都表现出中等的催化活性, 证 明了该反应可能经历了 Lewis 酸类型的催化作用机理.

2014 年, 苯并三唑类化合物也可以应用于磷酰化 试剂中, 所得衍生物具有较相应磷酰氯更优越的化学稳 定性. Panda 和 Hall 课题组 ${ }^{[61]}$ 研究发现, 苯并三唑类化 合物可以通过以下两种途径制备: (1)一锅两步法. 首先 亚磷酸二乙酯通过和次氯酸叔丁酯 $(t-\mathrm{BuOCl})$ 反应转化 为氯磷酸二乙酯中间体, 接着该化合物在碱性添加剂作 
用下和苯并三唑 $(\mathrm{BtH})$ 反应得到衍生化产物, 产率可达 92\% (Scheme 36, Path A). (2)一锅一步法. 在碱和氯代苯 并三唑的共同作用下, 亚磷酸二乙酯可以直接转化为相 应衍生物, 产率为 75\% (Scheme 36, Path B). 在碱性添 加剂或者微波(MW)反应器等辅助条件下, 该试剂表现 出非常高的反应活性, 苯并三唑也表现出较好的离去性 能，不但可以高效制备芳基、烷基磷酸混酯(Scheme 37), 还可以应用于含氮、硫等亲核试剂的高效转化, 为 $\mathrm{P}-$ $\mathrm{O}, \mathrm{P}-\mathrm{O}$ 以及 $\mathrm{P}-\mathrm{S}$ 键的构筑提供了新的思路和方法.

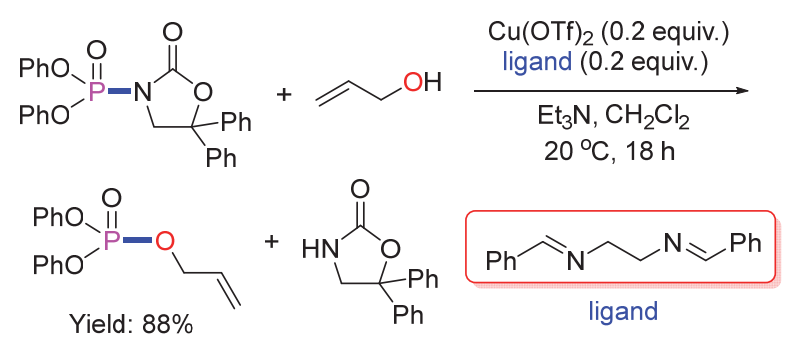

图式 35 含有噁唑烷酮的新型磷酰化试剂在铜盐作用下的催 化转化

Scheme 35 Catalytic conversion of a novel phosphorylation reagent containing oxazolidinone under the action of copper salt

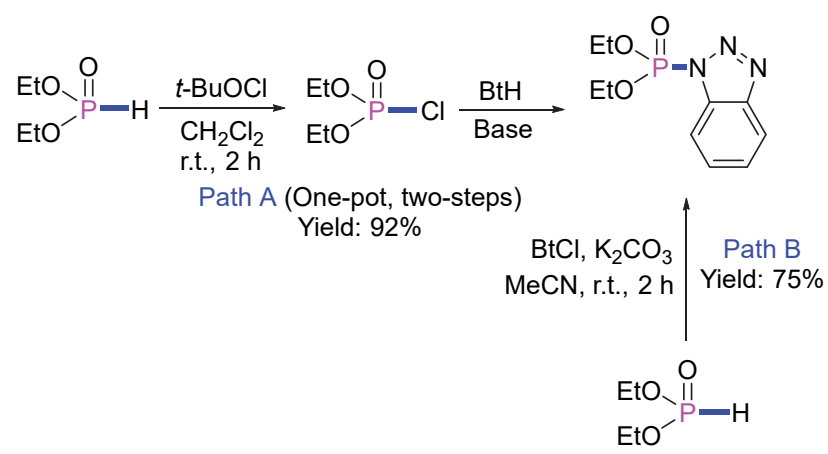

图式 36 苯并三坐类磷酰化试剂的转化方案

Scheme 36 Conversion scheme of benzotriazole phosphorylation reagent
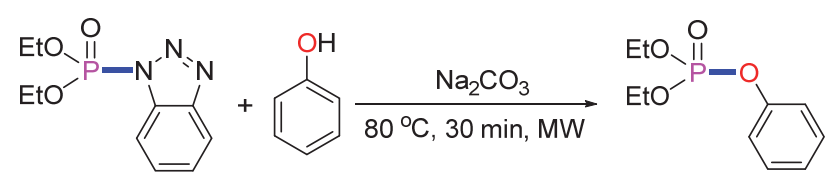

Yield: $77 \%$

图式 37 苯并三唑类磷酰化试剂在磷酸混酯合成中的应用 Scheme 37 Application of benzotriazole phosphorylation reagents in the synthesis of mixed phosphates

除了含有 $\mathrm{P}-\mathrm{N}$ 键官能团的磷酰化试剂之外, 作为 含有 $\mathrm{P}-\mathrm{C}$ 键的经典有机磷试剂, 氧基膦酸二乙酯 $(\mathrm{DEPC})^{[62]}$ 也可以作为芳基、烷基磷酸混酯的合成起始 物, DEPC 的合成路线如 Scheme 38 所示 ${ }^{[63]}$. Guzmán 和 $\mathrm{Diaz}^{[64]}$ 报道了在冰水浴下, DEPC 可以快速和各种类型
的苯酚或葲酚发生酯化反应. 然而，该反应会副产剧毒 性物质 $\mathrm{HCN}$, 因此, 需要在反应体系中加入三乙胺 $\left(\mathrm{Et}_{3} \mathrm{~N}\right)$ 中和酸性副产物(Scheme 39), 同时, 也是由于上 述原因, DEPC 在磷酸酯合成中的应用鲜有报道.

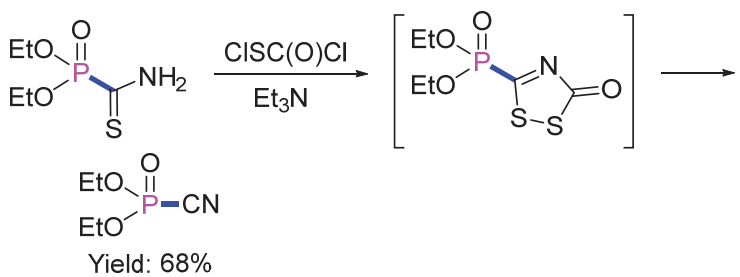

图式 $38 \mathrm{DEPC}$ 的合成路线

Scheme 38 Synthetic route of DEPC

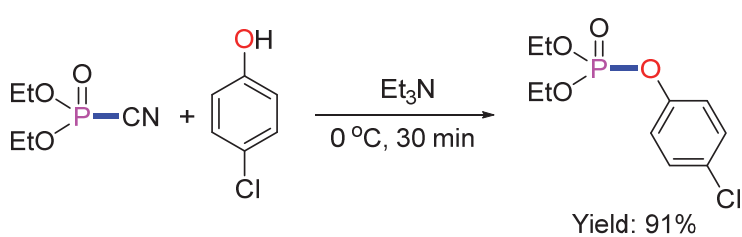

图式 39 氰基膦酸二乙酯与对氯苯酚的酯化反应 Scheme 39 Esterification reaction of diethyl cyanophosphonate with $p$-chlorophenol

\section{8 结论与展望}

综上所述，在过去一段时间里，新型的芳基、烷基 磷酸混酯类化合物的制备方法的开发受到了合成化学 和催化领域的广泛关注，已经从最初的使用较为苛刻的 反应条件、化学计量的促进剂、腐蚀性和不稳定性的起 始物发展成为构建更为简单和高效的催化体系，反应在 操作的简便性和起源原料来源的广泛性上都得到了一 定的发展. 近些年来，随着新型合成方法和技术的不断 涌现, 如光催化、电催化、压电催化、生物酶催化, 固 相研磨技术以及流动合成方法等，为科研人员提供了更 广泛、更理想的选择方案，也为制备方法的顺利放大提 供了更多可能. 希望本文能够有助于读者加深对该领域 的了解和动态掌握，并起到抛砖引玉的作用，使更多科 研工作者参与其中, 为我国磷化学的发展贡献力量.

\section{References}

[1] Kozak, W.; Rachon, J.; Daśko, M.; Demkowicz, S. Asian J. Org. Chem. 2018, 7, 314.

[2] Desloges, W.; Neverov, A. A.; Brown, R. S. Inorg. Chem. 2004, 43, 6752.

[3] Timperley, C. M.; Casey, K. E.; Notman, S.; Sellers, D. J.; Williams, N. E.; Williams, N. H.; Williams, G. R. J. Fluorine Chem. 2006, 127,1554

[4] Selikhov, A. N.; Malysheva, Y. B.; Nyuchev, A. V.; Sitnikov, N. S.; Sharonova, E. A.; Shavyrin, A. S.; Combes, S.; Fedorov, A. Y. Russ. Chem. Bull. 2011, 60, 2003.

[5] Mills, S. J.; Dozol, H.; Vandeput, F.; Backers, K.; Woodman, T.; Erneux, C.; Spiess, B.; Potter, B. V. L. ChemBioChem 2006, 7, 1696. 
[6] Silverberg, L. J.; Dillon, J. L.; Vemishetti, P. Tetrahedron Lett. 1996, 37, 771 .

[7] Jiao, L.-Y.; Zhang, Z.; Yin, X.-M.; Li, Z.; Ma, X.-X. J. Catal. 2019, $379,39$.

[8] Shen, J.; Zhang, Y.; Yu, N.; Crump, D.; Li, J.; Su, H.; Letcher, R. J.; Su, G. Environ. Sci. Technol. 2019, 53, 2151.

[9] Xu, D. CN 103360606, 2013.

[10] Liu, W. CN 109912646, 2021.

[11] Liang, R. CN 105833790, 2020.

[12] Stubbings, W. A.; Riddell, N.; Chittim, B.; Venier, M. Environ. Sci. Technol. Lett. 2017, 4, 292.

[13] Atherton, F. R.; Openshaw, H. T.; Todd, A. R. J. Chem. Soc. 1945, 660.

[14] Atherton, F. R.; Todd, A. R. J. Chem. Soc. 1947, 674.

[15] Cao, S.; Zhao, Y. Sci. China Chem. 2015, 45, 283 (in Chinese). (曹书霞, 赵玉芬, 中国科学, 化学, 2015, 45, 283.)

[16] Le Corre, S. S.; Berchel, M.; Couthon-Gourvès, H.; Haelters, J.-P.; Jaffrès, P.-A. Beilstein J. Org. Chem. 2014, 10, 1166.

[17] Chen, X.; Yu, Y.; Qu, L.; Liao, X.; Zhao, Y. Synth. Commun. 2004, 34, 493.

[18] Yang, Y.; Qu, C.; Chen, X.; Sun, K.; Qu, L.; Bi, W.; Hu, H.; Li, R.; Jing, C.; Wei, D.; Wei, S.; Sun, Y.; Liu, H.; Zhao, Y. Org. Lett. $\mathbf{2 0 1 7}, 19,5864$

[19] Okamoto, Y.; Kusano, T.; Takamuku, S. Bull. Chem. Soc. Jpn. 1988, 61, 3359 .

[20] Gupta, A. K.; Acharya, J.; Dubey, D. K.; Kaushik, M. P. Synth. Commun. 2007, 37, 3403.

[21] Anitha, T.; Ashalu, K. C.; Sandeep, M.; Mohd, A.; Wencel-Delord, J.; Colobert, F.; Reddy, K. R. Eur. J. Org. Chem. 2019, 7463.

[22] Wu, J.; Wang, B. Zhejian Chem. Ind. 2008, 39, 27 (in Chinese). (吴晶, 王博, 浙江化工, 2008, 39, 27.)

[23] Dhawan, B.; Redmore, D. J. Org. Chem. 1986, 51, 179.

[24] Powles, N.; Atherton, J.; Page, M. I. Org. Biomol. Chem. 2012, 10, 5940.

[25] Jones, S.; Selitsianos, D. Org. Lett. 2002, 4, 3671.

[26] Jones, S.; Selitsianos, D.; Thompson, K. J.; Toms, S. M. J. Org. Chem. 2003, 68, 5211

[27] Acharya, J.; Shakya, P. D.; Pardasani, D.; Palit, M.; Dubey, D. K.; Gupta, A. K. J. Chem. Res. 2005, 194.

[28] Liu, C.-Y.; Pawar, V. D.; Kao, J.-Q.; Chen, C.-T. Adv. Synth. Catal. $\mathbf{2 0 1 0}, 352,188$

[29] Xiao, P.; Zhang, J.; Feng, Y.; Wu, J.; He, J.; Zhang, J. Cellulose 2014, 21, 2369

[30] Weiss-Shtofman, M.; Kramer, M.; Dobrovetsky, R.; Portno, M. Org. Lett. 2020, 22, 3722

[31] Vignola, N.; Dahmen, S.; Enders, D.; Bräse, S. J. Comb. Chem. 2003, 5,138 .

[32] Sathe, M.; Gupta, A. K.; Kaushik, M. P. Tetrahedron Lett. 2006, 47, 3107.

[33] Gupta, A. K.; Kumar, R.; Dubey, D. K.; Kaushik, M. P. J. Chem. Res. 2007, 328

[34] Kasemsuknimit, A.; Satyender, A.; Chavasiri, W.; Jang, D. O. Bull.
Korean Chem. Soc. 2011, 32, 3486.

[35] Xiong, B.; Ye, Q.; Feng, X.; Zhu, L.; Chen, T.; Zhou, Y.; Au, C.-T.; Yin, S.-F. Tetrahedron. 2014, 70, 9057.

[36] (a) Xiong, B.; Feng, X.; Zhu, L.; Chen, T.; Zhou, Y.; Au, C.-T.; Yin, S.-F. ACS Catal. 2015, 5, 537.

(b) Xiong, B.; Zeng, K.; Zhang, S.; Zhou, Y.; Au, C.-T.; Yin, S.-F. Tetrahedron 2015, 71, 9293.

[37] Xiong, B.; Cheng, Q.; Hu, C.; Zhang, P.; Liu, Y.; Tang, K. ChemistrySelect 2017, 2, 6891.

[38] Xiong, B.; Hu, C.; Li, H.; Zhou, C.; Zhang, P.; Liu, Y.; Tang, K. Tetrahedron Lett. 2017, 58, 2482.

[39] Xiong, B.; Hu, C.; Gu, J.; Yang, C.; Zhang, P.; Liu, Y.; Tang, K. ChemistrySelect 2017, 2, 3376.

[40] Xiong, B.; Wang, G.; Zhou, C.; Liu, Y.; Li, J.; Zhang, P.; Tang, K. Phosphorus, Sulfur Silicon Relat. Elem. 2018, 193, 239.

[41] Bartlett, P. D.; Lonzetta, C. M.; J. Am. Chem. Soc. 1983, 105, 1984.

[42] Stephenson, L. M.; McClure, D. E. J. Am. Chem. Soc. 1973, 95, 3074.

[43] Oba, M.; Okada, Y.; Nishiyama, K.; Ando, W. Org. Lett. 2009, 11, 1879.

[44] Tamilselvi, A.; Mugesh, G. Chem.-Eur. J. 2010, 16, 8878.

[45] Kang, B.; Kurutz, J. W.; Youm, K.-T.; Totten, R. K.; Hupp, J. T.; Nguyen, S. T. Chem. Sci. 2012, 3, 1938.

[46] Totten, R. K.; Ryan, P.; Kang, B.; Lee, S. J.; Broadbelt, L. J.; Snurr, R. Q.; Hupp, J. T.; Nguyen, S. T. Chem. Commun. 2012, 48, 4178.

[47] Katz, M. J.; Mondloch, J. E.; Totten, R. K.; Park, J. K.; Nguyen, S. T.; Farha, O. K.; Hupp, J. T. Angew. Chem. Int. Ed. 2014, 53, 497.

[48] Huang, H.; Ash, J.; Kang, J. Y. Org. Lett. 2018, 20, 4938.

[49] Nilsson, J.; Kraszewski, A.; Stawinski, J. J. Chem. Soc., Perkin Trans. 2 2001, 2263.

[50] Kaboudin, B.; Mostafalu, R. Phosphorus, Sulfur Silicon Relat. Elem. 2012, 187, 776.

[51] Keith, J. M. J. Org. Chem. 2006, 71, 9540.

[52] Kim, H.; Park, J.; Kim, J. G.; Chang, S. Org. Lett. 2014, 16, 5466.

[53] Yamada, K.; Yamashita, M.; Sumiyoshi, T.; Nishimura, K.; Tomioka, K. Org. Lett. 2009, 11, 1631.

[54] Haberhauer, G.; Rominger, F. Eur. J. Org. Chem. 2003, 3209.

[55] Ying, J.; Gao, Q.; Wu, X.-F. Chem. Asian J. 2020, 15, 1540.

[56] Jiao, L.-Y.; Zhang, Z.; Hong, Q.; Ning, Z.-H.; Liu, S.; Sun, M.; Hao, Q.; Xu, L.; Li, Z.; Ma, X.-X. Mol. Catal. 2020, 494, 111120.

[57] Matsumoto, S.; Masuda, H.; Iwata, K.; Mitsunobu, O. Tetrahedron Lett. 1973, 14, 1733.

[58] Maezaki, N.; Furusawa, A.; Hirose, Y.; Uchida, S.; Tanaka, T. Tetrahedron 2002, 58, 3493.

[59] Jones, S.; Smanmoo, C. Tetrahedron Lett. 2004, 45, 1585.

[60] Jones, S.; Smanmoo, C. Org. Lett. 2005, 7, 3271.

[61] Panmand, D. S.; Tiwari, A. D.; Panda, S. S.; Monbaliu, J.-C. M.; Beagle, L. K.; Asiri, A. M.; Stevens, C. V.; Steel, P. J.; Hall, C. D.; Katritzky, A. R. Tetrahedron Lett. 2014, 55, 5898.

[62] Harusawa, S.; Shioiri, T. Tetrahedron. 2016, 72, 8125.

[63] Łopusiński, A. Heteroat. Chem. 2004, 15, 395.

[64] Guzmán, A.; Diaz, E. Synth. Commun. 1997, 27, 3035. 